\title{
BINARY STAR RESEARCH: WHERE WE ARE NOW AND FUTURE PROSPECTS
}

\author{
E. F. GUINAN \\ Department of Astronomy \& Astrophysics \\ Villanova University \\ Villanova, PA USA
}

ABSTRACT: A review of IAU Symposium 151 on Interacting Binary Stars is given along with discussions of the present and future prospects of research in close binary stars.

\section{Introduction}

As indicated by the high quality and large quantity of the papers presented at IAU Symposium 151 in Cordoba, interest and research in interacting binaries continues to prosper and grow. At this conference, I think every conceivable class of interacting binary was represented in the oral presentations or poster formats. These binaries included every type of stellar object from pre-mainsequence and main-sequence stars to giants and supergiants as well as the degenerate end products of stellar evolution - white dwarfs, neutron stars and black holes. In addition to stars or stellar fossils, many binary systems have accretion disks and were featured in several papers at this meeting.

The study of interacting binaries provides insights into nearly all areas of astrophysics - including stellar interiors and atmospheres, stellar evolution, nucleosynthesis, plasma physics, magnetic dynamos (in cool stars), and relativistic physics to name a partial list. Recently, the study of eclipsing binaries in other galaxies now makes it possible to explore stellar structure and evolution and establish mass-luminosity laws for galaxies with vastly different evolutionary and chemical histories from our Galaxy (such as the LMC and SMC). Moreover, as first presented at this conference (see Bell et al. and Niemela and Bassino), eclipsing binaries are beginning to play an important role in cosmology as primary distance indicators to nearby galaxies. As more data are accumulated, the studies of these systems may lead to an improvement in the extragalactic distance scale. Some day in the not too distant future there may be symposium devoted to extragalactic interacting binaries!

\section{Some Important New Results From IAU Symposium 151}

- Theoretically, it is still not possible to make close binaries. According to Peter Bodenheimer, there are major theoretical problems with the formation of close binary systems.

- But close binaries do exist!

- Jorge Sahade presented a tour de force discussion of the known species of interacting binaries and the physical processes occurring in each type. He recommends more intensive multifrequency observations and complete orbital coverage to understand them.

- Be Stars, however, are not close binaries (see Dietrich Baade's discussion).

- Barium Stars, on the other hand, may all be members of binary systems containing white dwarf components, but many of the white dwarfs are too faint to detect. (Verne Smith). 
- Peter Eggleton introduced the Theoretical Bright Star Catalogue (TBSC) and discussed mass loss and transfer in close binaries. According to his calculations, Barium stars should be more common than Algol systems. In fact, about 23 Barium stars with white dwarf companions are found in the $T B S C$, but this finding conflicts with the single Barium star/white dwarf pair found in the actual Bright Star Catalogue. The question was raised whether Sirius A should be enriched with Barium and other heavy metals from the progenitor of its white dwarf companion. The lack of such evidence for chemical pollution for Sirius A results from the Sirius $\mathrm{AB}$ orbit probably being too wide to permit significant mass accretion. Eggleton is continuing his theoretical study of bright stars in the TBSC. Several observational astronomers requested finding charts and coordinates for the $T B S C$ stars.

- All technetium-deficient $S$ and MS stars probably are mass-transfer binaries. The mass transfer, however, may have taken place while the current $M$-star was on the main-sequence ( Hollis Johnson).

- As demonstrated by several papers presented at this meeting, eclipsing binaries still play a crucial role in stellar astrophysics. Eclipsing binaries are rich sources of vital information on the physical properties of stars (masses, radii, luminosity and internal structure in systems that show apsidal motion). Eclipsing systems (such as the $\zeta$ Aur binaries) provide important opportunities to probe the outer atmospheres of cool giant and supergiant stars. (For significant examples see Giménez and Carpenter.)

- As shown in a number of talks and papers, close binary systems with cool components provide important testing grounds for studying high levels of presumably dynamo-generated magnetic activity. These binaries include W UMa, RS CVn, BY Dra, and Algol systems. Also, magnetic fields play an increasingly appreciated role in the evolution of binaries through angular momentum loss via magnetic braking by stellar winds (discussions by Rodono, Vilhu and others). As discussed by David Henemoerder, the rapidly rotating G-type giant, FK Comae, still remains a viable candidate of the recent coalescence of a low mass close binary.

- A decade ago we had little firm observational evidence of pre-main-sequence (PMS) binaries. As shown by Mathieu, the discovery rate of PMS binary systems has dramatically increased over the past decade. Spectroscopic, occultation, speckle interferometry, as well as other techniques have found PMS binaries at all separations and at a wide range of ages. Masses derived from spectroscopic observations for the first time permit observational checks on PMS evolutionary tracks. Pre-main-sequence binary frequencies appear comparable to full main-sequence frequencies except among the classical $\mathrm{T}$ Tauri stars where the number of detectable binary systems appears abnormally low.

- Mass transfer in interacting binaries with white dwarf components are responsible for a significant fraction of supernova explosions as the white dwarf accretes to the Chrandrasekhar limit. Several symbiotic stars have overweight white dwarf components $\left(m \approx 1-1.2 \mathrm{M}_{\odot}\right)$ that could with additional mass gain reach critical mass and become supernovae. (See e.g., discussions by Craig Wheeler, Scott Kenyon, Yasuo Tanaka and Harry Nussbaumer.)

- Accretion disks are fairly common in many types of binaries and include the relatively large, extended cool accretion disks associated with pre-main-sequence binaries, and the long period Algol binaries and many symbiotic stars. On the other hand, accretion disks evident around the white dwarf components of CVs are more compact and hotter $\left(\mathrm{T}_{\max } \geq 10^{5}-10^{6} \mathrm{~K}\right)$, while the accretion disks associated with the neutron star and black hole components of X-ray binaries are typically compact and extremely hot $\left(\mathrm{T}_{\max } \geq 10^{7} \mathrm{~K}\right)$. Observations and theoretical studies of accretion disks and accretion processes were discussed in some detail by several of the participants, including Smak, Kaitchuk, Guinan and King. 
- The flip-flop model was discussed by Mario Livio in explaining the behavior of wind-accreting binaries with disks. The flip-flopping may be due to disk instabilities and depends on the direction in which the accreting matter enters the disk.

- Mass transfer in interacting binaries appears to be far from conservative (e.g., J.-P. DeGréve) and a great deal of gas can be lost from close binaries to the interstellar medium. This mass loss which can be quite significant (up to several solar masses) will pollute (or enrich if you like) the interstellar medium (ISM), quite often with gas processed by thermonuclear reactions see (Polidan). Alan Batten was one of the first to point out this important aspect of interacting binaries which hitherto was largely overlooked. Thus, interacting binaries may modify (and heat) the ISM and affect the evolution of the galaxy in which they reside.

- One of the most exciting developments over the last decade in the field of binary star research is our capability of obtaining accurate radial velocity and light curves of binaries in other galaxies (see papers by Bell et al. and Niemela and Bassino). This breakthrough occurred chiefly because of the development of high quantum efficiency/low noise CCDs. At this conference, Bell et al. report an accurate distance determination to the LMC using an eclipsing binary. This determination is in good agreement with the distance determined using other methods. Also, observations of binary stars in Galactic and Globular Clusters is leading to a better understanding of binary star evolution and cluster dynamics. See discussions by Trimble, Milone, Leung, and Stagg.

- Danniel Popper has identified an interesting small group of semi-detached systems (RT Lac for one) having detached components with spectral types of $\mathrm{G}$ or $\mathrm{K}$. He has named these systems Cool Algols. An alternative name for the group could be semi-detached RS CVn binaries since the group has high levels of chromospheric activity (e.g., strong $\mathrm{Ca}$ II $\mathrm{H}+\mathrm{K}$ emission). The Cool Algols form a sequence having temperature decreasing with increasing mass - a contrast to the classical Algols. The detached components of these binaries have properties (M, R, T, L) similar to those of detached MS stars.

- Bursters have been tentatively explained by Tanaka to be produced by accretion flow onto neutron stars. He shows this accretion to be quite unstable leading to sudden outbursts of $\mathrm{X}$-rays. About 10 observed transients are expected to be black holes in binary systems. Extrapolating to the entire Galaxy, he estimates about 100-1000 black hole binary systems, most of which will be $\mathrm{X}$-ray quiet and difficult to detect.

- In a paper presented by Virginia Trimble on behalf of Joseph Taylor, it appears that binary pulsars are: 1) Born Great, i.e., formed from the evolution of relatively massive stars in wide binary systems; (2) Achieve Greatness, by a white dwarf component accreting enough mass to reach the Chrandrasekhar limit; or (3) Have Greatness Thrust Upon Them by capture/collision exchanges that are possible in the star rich cores of Globular Clusters. Radio surveys now indicate about 28 radio pulsars in Globular Clusters. M13 and 47 Tuc are each rich in radio pulsars indicating that captures might be important. And there is strong evidence that binary millisecond pulsars are recycled pulsars.

- Every new X-ray satellite has discovered a new class of X-ray binary. A comprehensive review of the different species of X-ray binaries was given by Virginia Trimble. Everything, from Low Mass X-ray Binaries (LMXRBs), High Mass X-ray Binaries (HMXRBs), Be/neutron star systems, Black Hole Binaries, $\gamma$-ray Bursters, and $\mathrm{TeV}$ and $\mathrm{BeV}$ objects to the exotic binary SS433, was discussed. Joe Smak raised the interesting question: Why are there no neutron stars with masses greater than $\approx 1.4 \mathrm{M}_{\odot}$ ? This remains an important observational and theoretical question. 
- Other interesting topics include close-binary nuclei of planetary nebulae (Bond), Wolf-Rayet (WR) systems (Cherepashchuk) and a discussion of colliding winds in hot, massive binaries (Shore). The $10^{\text {th }} \mathrm{mag}$, former eclipsing binary SS Lac no longer shows eclipses (see Schiller and Milone)!

\section{Astrophysical Importance of Different Classes of Close Binaries}

There is not enough time in this brief review to discuss the various classes and subclasses of interacting binaries that can and do occur in nature. I have tried in Figure 1 to include most of the broad classes of interacting binaries that were discussed at this symposium. I have also included the important physical processes taking place in very broad terms and some examples. Binary systems divide broadly into those containing at least one degenerate (white dwarf, subdwarf, neutron star or black hole) component and those consisting of two non-degenerate stars. The binary classes are also plotted against orbital period and the approximate ranges of orbital periods for each class. No attempt was made to impose evolution onto the diagram, but, for the most part, the evolutionary time arrow in the diagram is downward. Moreover, many non-interacting binaries and some astrometric binaries will eventually evolve into interacting systems - depending on their initial separation and the masses of the component stars.

Below, I briefly discuss the main types of interacting binaries with some reasons why these systems are interesting or important. Membership in a binary where interaction occurs (mass transfer, mass loss, accretion, tidal interaction, etc., ) leads some binary stars to become supernovae while other binaries may merge to become single stars.

3.1 DETACHED ECLIPSING BINARIES: Provide fundamental data on stellar masses, radii, luminosity, and limb darkening. Also, from systems showing apsidal motion, the internal mass distribution can be determined.

3.2 SEMI-DETACHED BINARIES: A. Classical Algols: Provide checks on stellar properties and evolution as well as yield information on mass loss and mass exchange. B. Active Algols (The $W$ Ser Binaries): Provide laboratories for studying rapid (short-lived) stages of stellar and binary star evolution. The systems are important for studying accretion processes and accretion disks. Mass loss from these systems may contribute to the chemical enrichment of the interstellar medium (ISM).

3.3 RS CVn and BY Dra BINARIES (= Chromospherically Active [CA] Binaries): Important systems for investigating dynamo driven, magnetic activity in cool stars (spectral type $F$ and later). Manifestations of enhanced magnetic activity include: starspots, chromospheres and coronae as well as flares. These systems are important for learning about magnetic activity of the Sun - the so-called solar-stellar connection.

$3.4 \mathrm{~W}$ UMa CONTACT SYSTEMS: These short period $\left(0 \stackrel{d}{\cdot} 2 \leq P \simeq 0^{d} \cdot 8\right)$ contact/overcontact binaries display very high levels of magnetic activity and are important stars for studying the stellar dynamo at extreme (maybe saturated) levels. Magnetic braking may cause these binaries to coalesce into single stars (i.e., FK Comae stars/blue stragglers).

3.5 CATACLYSMIC VARIABLE (CV) AND NOVA-LIKE (NL) BINARIES: These short period systems contain white dwarf components and cool M-type secondaries which fill their Roche lobes. CVs and NLs provide valuable information on the final stages of stellar evolution. Also, these binaries are important for studying accretion phenomena and accretion disk properties.

3.6 X-RAY BINARIES WITH NEUTRON STAR AND BLACK HOLE COMPONENTS (LMXRBs, HMXRBs and Related Systems):These systems are powerful $\left(\mathrm{L}_{x}>10^{35}\right.$ ergs s$\left.^{-1}\right) \mathrm{X}$-ray sources 
which have neutron star or (more rarely) black hole components. The X-rays arise from accretion of gas onto the degenerate component of the system from a non-degenerate companion. Observations of these systems supplement the information on neutron star structure and evolution that comes from pulsars such as masses, radii, rotation, magnetic fields. Systems such as Cyg X-1 provide possible proofs of the existence of black holes.

3.7 $\zeta$ AURIGAE AND VV CEPHEI SYSTEMS: These long period interacting binaries contain a G$\mathrm{M}$ supergiant component and a hot (usually B-spectral type) companion. These systems originally were not interacting binaries but became so when the more massive star evolved to become a supergiant. They are important systems when eclipses occur because the atmospheric structure of the cooler supergiant can be probed as the hotter component passes behind it prior to and after primary eclipse. $\zeta$ Aur systems contain G-K supergiant stars while VV Cep binaries contain $\mathbf{M}$ supergiants.

3.8 SYMBIOTIC BINARIES: Symbiotic stars are long period interacting binaries which consist of a M-giant (sometimes a pulsating Mira-type variable) and an accreting component which can be a white dwarf, subdwarf, or low mass main sequence star. $\zeta$ And is frequently referred as the prototype of this class but, as discussed by Kenyon, their properties vary greatly from star to star. The common trait, however, is accretion of the cool component's wind by its hot companion. Orbital periods of symbiotic stars typically range between $\approx 200-1500$ days. Several of the symbiotic binaries have the cool component filling their Roche lobes - making them Symbiotic Algol systems (e.g., T $\mathrm{CrB})$.

3.9 BARIUM AND S-STAR BINARIES: These stars are now thought to be long-period binaries in which the originally more massive component evolved and transferred some its nuclear processed gas to the present K-M giant component. These stars are thought to have white dwarf (often too cool to be seen in the UV) companions. They are important for studying nucleosynthesis and mass loss in evolved stars.

3.10 POST COMMON ENVELOPE BINARIES: These binary systems usually contain hot white dwarf or subdwarf components and cooler secondary stars that have presumably passed through the common envelope phase of binary star evolution. The binary nuclei of planetary nebula are examples of Post CE Binaries discussed by Bond at this meeting. An additional well-known example is the $9^{\text {th }}$ mag Hyades eclipsing binary V471 Tau (DA2 $\left.+\mathrm{K} 2 \mathrm{~V} ; \mathrm{P}=0^{d} \cdot 52\right)$. V471 Tau and other related systems are important for studying short-lived stages of stellar evolution.

\section{New Directions and Opportunities}

As we approach the end of the $20^{\text {th }}$ century, the future looks bright for work on interacting binaries. New directions and opportunities for research in close binaries are already apparent as new technologies and telescopes become available on the ground and in space. In the next few years, many important binary systems which have been hitherto too faint or difficult for study with existing telescopes and equipment will be (and even now are) accessible for study over the entire electromagnetic spectrum, from $\gamma$-rays to radio wavelengths. On the theoretical side, bigger and faster computers and their increasing availability are are making it possible to construct more realistic physical models (with fewer approximations and limitations) for binary systems and the evolutionary and dynamic processes taking place in them. The following sections give a few highlights of the progress now being made.

4.1 AUTOMATIC PHOTOMETRIC TELESCOPES (APTs): During the last few years automatic photometric telescopes (APTs) have become a very reliable and efficient means of securing wellcovered, high precision light curves of close binaries down to $\approx 11^{\text {th }}$ magnitude. Moreover, the 
$A P T$ works on while the astronomer sleeps and doesn't mind observing on holidays or anniversaries or even in cold weather. At present, most of the operating APTs are located in the Southwest of the USA. Recently, an $0.75 \mathrm{~m}$ APT began operation in Catania, Italy and several more are being built in Europe, Korea and Australia. Plans are being made to establish a global network of $0.75 \mathrm{~m}$ APTs (GNAT: Global Network of Automatic Telescopes) so that continuous coverage of interacting binaries and other types of variable stars can be conducted. Photometry would be carried out with the same telescopes, filters, detectors, etc., , worldwide, eliminating problems of combining observations obtained with different telescopes and instrumentation. the GNAT system would also make it possible to obtain complete light curves of some difficult systems. Binaries with integer day periods or rapidly changing light curves such as RS CVn systems, cataclysmic binaries and Algol stars would benefit from a GNAT system. At this meeting interesting results were presented on the Whole Earth Telescope - a global network of non-automatic telescopes that is capable of continuously monitoring stars over a several day period. In the future we will expect comparable results from the GNAT system as it develops.

4.2 NEW GENERATION DETECTORS (CCDs): Also, the increasing availability of low cost, high quantum efficiency CCDs has had a strong impact on the study of close binaries. This is demonstrated at this conference by papers based directly or indirectly on spectroscopy or photometry with CCD detectors. For example, CCD photometry has permitted high precision $B$ and $V$ light curves to be obtained on some faint $\left(15^{\text {th }}-18^{\text {th }} \mathrm{mag}\right)$ eclipsing binary members of star clusters as well as the Large and Small Magellanic Clouds (LMC and SMC). These observations were carried out on moderate-aperture (1-2m) telescopes. Spectrographs equipped with CCDs have also permitted many formerly single-line spectroscopic binaries to be resolved into double-line systems - especially with the use of cross-correlation techniques. CCD spectroscopy has also permitted excellent spectra to be obtained of faint but important binaries such as CVs and faint X-ray binaries. Many of the papers at this meeting would not have been possible without these highly efficient detectors. Unfortunately, with quantum efficiencies of up to $80 \%$, the sensitivity of these CCDs is approaching its limit. However, the signal-to-noise resolution (smaller pixels) and the increasing area of the chips (more pixels) are increasing while the costs of these devices are decreasing.

4.3 RADIO OBSERVATIONS: The Very Long Array (VLA) and the Very Long Baseline Interferometer (VLBI) are providing crucial information about the radio emission of different types of interacting binaries - from binary pulsars to chromospherically-active binaries. For example, ultraprecise differential astrometry with positional accuracies of $\leq 1$ milliarc sec $(=0.001)$ are now possible with the $V L B I$. This has led to the astrometric identification of the $\mathrm{cm}$-radio source in the Algol system (by Lestrade and collaborators). Continued improvement of radio facilities and international cooperation should lead to radio positions and detailed radio maps of interacting binaries.

4.4 SATELLITES: In addition to these developments in ground-based optical, infrared, and radio astronomy, the era of orbiting astronomical observatories has had a radical influence on the developing understanding of interacting binaries. X-ray satellites such as EINSTEIN, Ginga, EXOSAT, and, most recently, ROSAT have had an enormous impact on the field of binaries with compact components (such as CVs and X-ray binaries) and on the study of solar-type coronal sources such as RS CVn, Algol, and W UMa systems. ROSAT is the only X-ray satellite now operational and it is expected to continue functioning until 1994/5. Astro-D is the Japanese successor to Ginga and its launching is planned for 1993, while the X-ray Timing Explorer (XTE) and the Advanced X-ray Astrophysical Facility (AXAF) should be in orbit before the end of the decade. These satellites will greatly expand our knowledge about X-ray binaries and coronal X-ray sources.

The International Ultraviolet Explorer (IUE) satellite, launched in 1978 and still going strong, probably has had the largest impact on the field of interacting binaries as well as on numerous 
other areas of stellar and extragalactic astrophysics. As IUE goes into its $15^{\text {th }}$ year of operation, important contributions to close binaries continue to be made. IUE has been especially important in providing time-critical and continuous observations over the wavelength range of $\lambda \lambda 1150-3200$ because of its high, geosynchronous orbit. IUE observations have created a renaissance in binary studies, leading to a better understanding of the complex dynamical and evolutionary processes in binary systems.

The Hubble Space Telescope (HST), although chiefly designed for extragalactic work, should also play an important role in interacting binaries - especially at UV wavelengths. Even with its present problems (spherical aberration, failure of the high resolution FUV spectrograph), the $H S T$ is yielding excellent, high-signal-to-noise spectra for many different classes of binary stars. Because of its larger mirror and more sensitive and low noise detectors, HST is very useful for investigating faint X-ray binaries in our galaxy as well as in the LMC and SMC. Because of shorter exposure times, better time resolution is possible with $H S T$ relative to IUE and this will be important in studying short period systems such as CVs, LMXRBs and HMXRBs. Also, the high time resolution will be important for studying transient events (flares and outbursts) in chromospherically active binaries such as the W UMa, BY Dra and RS CVn stars. High signal-to-noise UV spectra will also be important in Doppler imaging of a star's active regions using density and pressure-sensitive chromospheric and transition region line emissions (such as the C III $\lambda 1909$ line).

In the Spring of 1992, the Extreme Ultraviolet Explorer (EUVE) satellite is planned for launch. This satellite has a wavelength range, straddling the X-ray and FUV regions, of 90-820 $\AA$. This mission, if successful, should have a major impact on studies of both interacting binaries with hot, degenerate components and those with hot disks, such as nearby CVs, AM Her, and novalike binaries. The satellite should also be effective in studying chromospheric transition region and coronal activity in nearby RS CVn systems. It should be noted that, because of the strong extinction of the EUV from the ISM, most of the targets will have to be nearby $(\mathrm{d} \leq 100 \mathrm{pc})$ except in directions where ISM is less dense.

The Gamma-Ray Observatory (GRO) was successfully deployed in 1991; it has an effective energy range of $\approx 50 \mathrm{KeV}$ to $30,000 \mathrm{MeV}$. This satellite should play a significant role in our understanding the nature of bursters as well as close binary systems with very hot neutron star and black hole components. Other planned space missions that will have a great impact on close binaries are the Advanced X-ray Astrophysics Facility (AXAF), the Space Infrared Telescope Facility (SIRTF) and the radio missions such as the International $V L B I$ satellite.

4.5 MOON-BASE FACILITY: Perhaps within the next 20 years, the study of binary stars may be done from an International Lunar Astronomy Base where, among other instrumentations, 10$20 \mathrm{~km}$ interferometric arrays could permit the spatial resolution of many close binary systems into astrometric binaries. More high resolution spatial maps of many complicated systems should permit these binaries to be directly imaged. It will be interesting to discover how close we are to understanding interacting binaries and, of course, where we have erred in our interpretations.

\section{Conclusions}

Symposium 151 was a most successful and enjoyable meeting. It was especially meaningful to have such distinguished senior colleagues as Alan Batten, Daniel Popper, Jorge Sahade and Brad Wood attend and contribute to the scientific content of this symposium. Their work in the field of interacting binaries over the past half-century and more has greatly advanced understanding of these stars. It is also gratifying to have so many younger astronomers participating in the meeting and studying interacting binaries. We look forward to their contributions as the new century dawns.

On behalf of all the attendees, I would like to thank Roberto Sistero and the members of the Local Organizing Committee for their help and hospitality. Finally, I hope that we are all around in the $21^{s t}$ century to enjoy the anticipated new and exciting discoveries in interacting binary studies! 


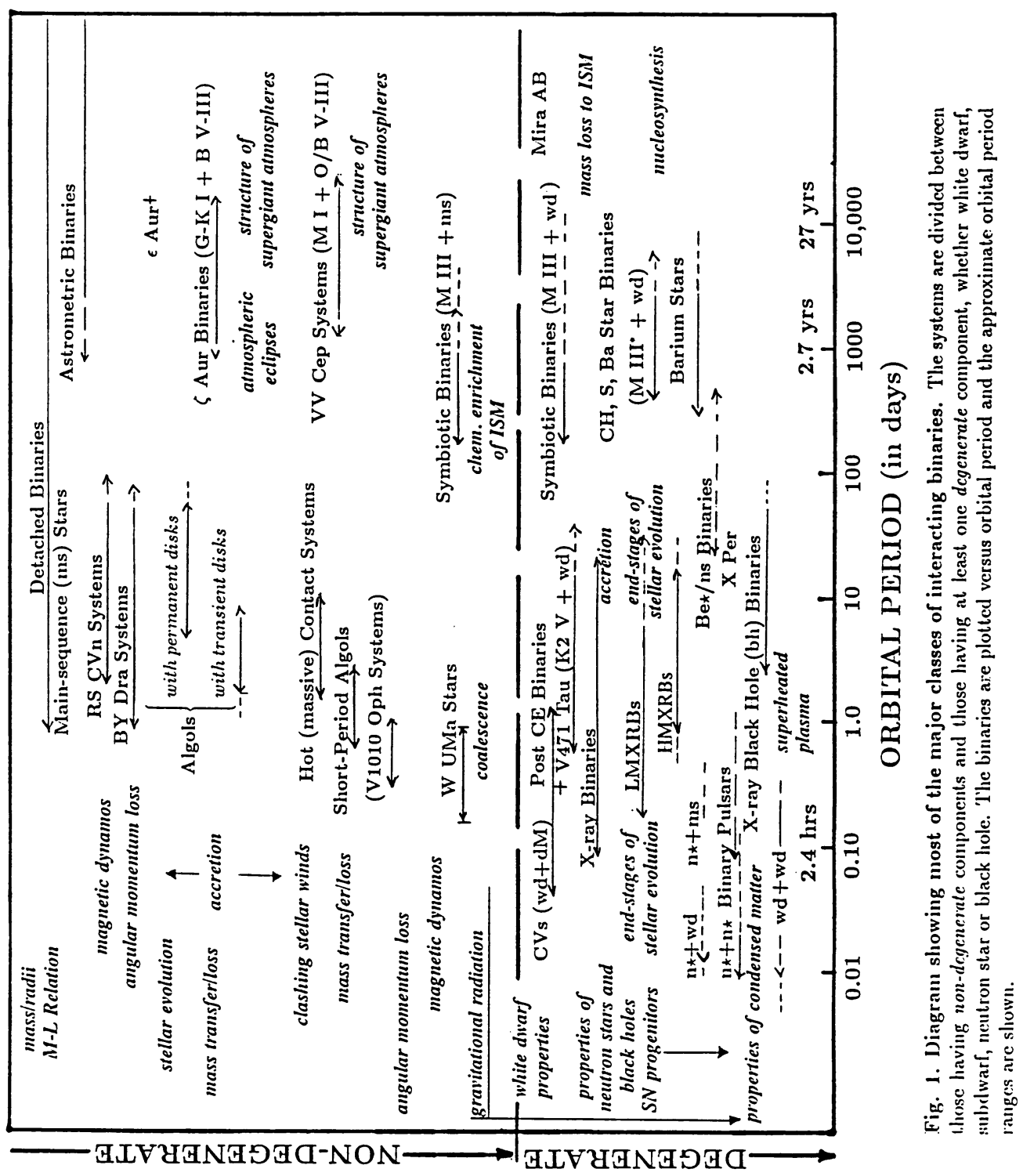

$\overline{\text { TdXI \&VLS }}$ 